\title{
Multimode Interference Structures of Variable Geometry for Optical Sensor Application
}

\begin{abstract}
A. SzewCZuK* AND M. BŁahut
Department of Optoelectronics, Silesian University of Technology, Krzywoustego 2, 44-100 Gliwice, Poland

The paper presents the analysis results of a waveguide sensor based on multimode interference structures. The multimode section was covered with sensor material. The change of optical parameters of the cover entails the change of propagation conditions of light in the structure. By measuring the light intensity at the output of the structure, we can define external physicochemical parameters to which the sensor layer is sensitive. The paper presents the method to adjust the sensitivity of the device through a proper selection of the thickness of the particular layers of multimode waveguides. We present, among others, the analysis results of optical systems whose parameters of the sensor layer corresponded with the parameters of wolfram oxide $\left(\mathrm{WO}_{3}\right)$, being the material frequently applied as a gas-sensitive layer in gas sensors. We have proposed a configuration of a sensor based on the Mach-Zehnder interferometer whereof one arm is a multimode section and the other one a single mode waveguide. The detection method was narrowed down here to the measurement of phase difference between the waves propagating in the respective arms.
\end{abstract}

PACS numbers: 42.79.Gn, 42.82.Ds, 42.79.-e

\section{Introduction}

Optical interference sensors have been for many years subject to intensive studies [1-3]. Recently, a growing interest in the multimode interference (MMI) structure application can be observed. There is a lot of research and analysis results published involving MMI devices designed as temperature [4] or chemical sensors/biosensors $[5,6]$. These sensors have many advantages such as simple operation, high sensitivity etc. The principle of operation of each of the different configurations described in many scientific publications is similar. In most cases, the multimode section is covered with a layer whose optical parameters are changed when exposed to a measured medium. Many investigation studies concerning the research on optical sensors based on the multimode interference coupler have been published. One of the first concepts was discussed in papers $[7,8]$. According to the presented results, these optical sensors allow to measure the changes of chemical parameters of the surrounding environment with a high sensitivity.

The main objective of the work was to elaborate and analyze the systems based on multimode interference structures which can find application as waveguide sensors. The analysis of the particular models was based on the numerical BPM method $[9,10]$. The analysis was focused on concepts where the energy of the excited modes was located principally in the core, and the interaction with the sensor layer was affected mainly through the

* corresponding author; e-mail: artur.szewczuk@polsl.pl evanescent field of the modes. Also, structures were modeled for which the light was guided through the modes excited in the sensor layer.

\section{Operation principle}

Multimode interference structures are generally produced from three waveguide sets. When exciting a multimode waveguide, we can observe the matching of input field to the modal fields of the waveguide, and then the interference of the produced waves. In effect of the intermodal interference, a so-called self-imaging of the input field is taking place. As a result of this effect, the input field is reproduced in simple, reflected and multiple images [11, 12].

The modeled sensor consisted of a single mode waveguide at the input and output, and a multimode waveguide was placed between them. The MMI section consisted of several layers. The basis was made up by a buffer layer whose aim was to isolate the core from the substrate. On the buffer layer, the core lined with the cover layer was placed. A sensor layer was built in into the cover and placed directly on the core.

When the refractive index of the sensor layer is changing in effect of the interaction with the external medium, the propagating conditions are changing and finally the output signal is getting changed.

\section{Optical structures}

In the paper we have proposed different sensor models. The differences involved geometrical dimensions and 
also the optical parameters of the modeled waveguides. We also investigated the system working in configuration with the Mach-Zehnder interferometer whereof one arm was made up by a multimode section. Making use of the BPM method $[6,11]$, the performance characteristics of the investigated configurations were presented.

\subsubsection{Design}

\subsection{Symmetrical MMI structure}

In the case of the first configuration, the thickness of the sensor layer was $0.5 \mu \mathrm{m}$ and that of the core was $4 \mu \mathrm{m}$. The refractive index of the substrate was 1.49 and that of the core was 1.5. For such parameters, for the wavelength of $1310 \mathrm{~nm}$, the modes could be excited both in the multimode core and in the sensor layer (Fig. 1). In this case, the interaction was affected through the evanescent field of the modes from the core layer and also through the field of the modes excited in the sensor layer (Fig. 2). During the simulation the refractive index was being changed within the range of $1.85-1.852$. The length of the multimode waveguide was defined in the way to ensure the acquisition of 1 -fold image of the input field at the output for the refractive index of the sensor layer of 1.85 .

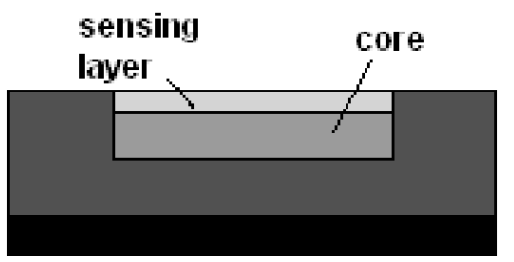

Fig. 1. Structure diagram.

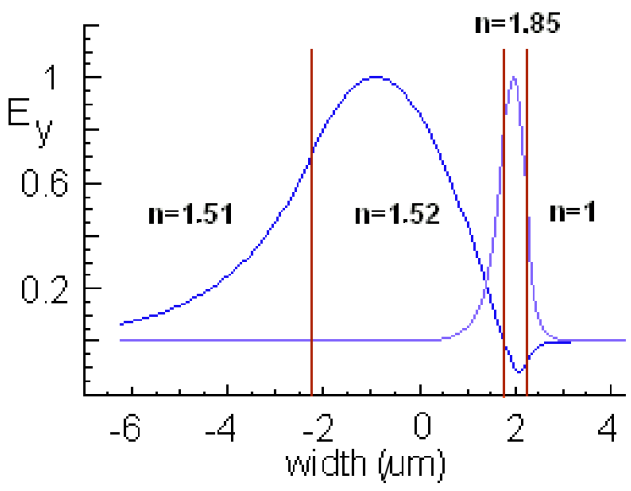

Fig. 2. Wave functions of the modes excited in the structure.

\subsubsection{Results and discussions}

Figure 3 presents the dependence of the field amplitude at the output of the structure as a function of refractive index of the sensor layer obtained on the basis of numerical calculations. The calculations were carried out for the modes of the multimode section width of 25,40 and $60 \mu \mathrm{m}$.

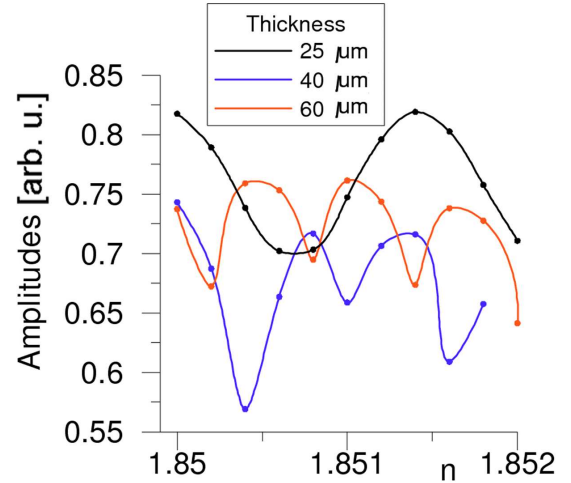

Fig. 3. Dependence of the output field amplitude as a function of refractive index of the cover.

The amplitude changes are here of oscillatory character. The oscillation period is connected with the width of the multimode section. The dynamics of changes for these types of structures is very high. The form of the presented dependence is conditioned by the influence of modes coupling from the sensor layer and from the core. The figure presents the field distribution in both layers (Fig. 4).

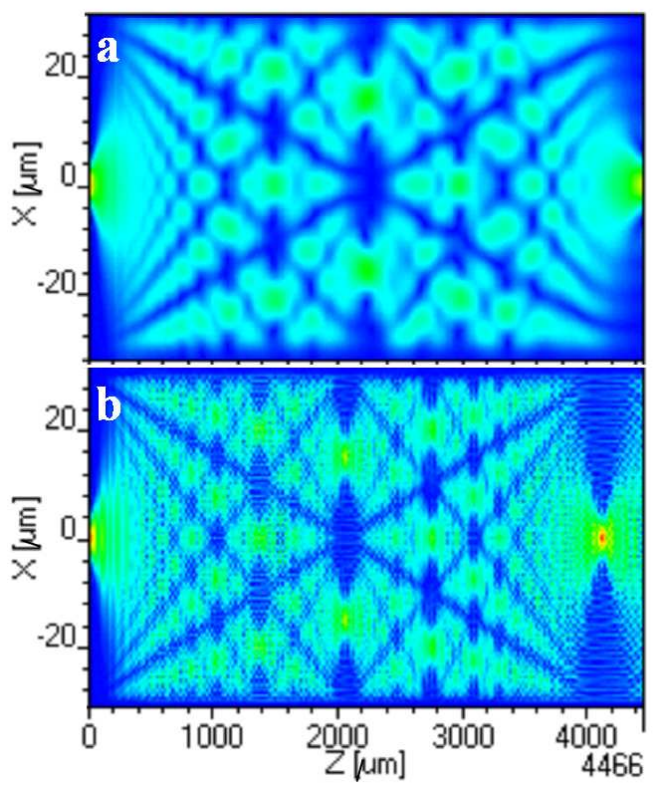

Fig. 4. Field distributions along the propagation path in the multimode core (a) and in the sensor layer (b).

The field distribution in the sensor layer is conditioned by the presence of intermodal interference of modes excited in this layer, and is different from the field in the core. The light propagating in the cover layer is also adjusting to the modal fields of this layer. Of course, at the same time there is a mutual interaction between the fields from both layers through the evanescent fields and the interference of waves from both waveguides. These 
factors have influence on the periodic shape of the characteristics from the figure (Fig. 4).

Another parameter which should be taken into consideration while describing the interaction of MMI structures with the cover layer is material extinction. This factor is particularly important for sensor layers used for gas detection, which will be described in the next part of the paper.

\subsection{Symmetrical MMI structure with the sensor layer based on $\mathrm{WO}_{3}$ material}

\subsubsection{Material aspects}

Metal oxides are commonly applied materials used as gas-sensitive layers in gas sensors. They are characterized by good sensitivity to such gases as: $\mathrm{CO}, \mathrm{H}_{2}, \mathrm{NO}_{x}$, $\mathrm{CO}_{2}, \mathrm{O}_{3}$ and hydrocarbons. The following metal oxides are most frequently applied in sensors: $\mathrm{SnO}_{2}, \mathrm{ZnO}$, $\mathrm{TiO}_{2}, \mathrm{In}_{2} \mathrm{O}_{3}, \mathrm{WO}_{3}$ [7]. For the analyzed concept, we modeled a sensor layer making use of the parameters of the $\mathrm{WO}_{3}$ material, being a popular sensor material applied in gas sensors. The parameters applied during the simulation were provided from the publication [6]. In this publication the authors present the research results which were to define the optical parameters of $\mathrm{WO}_{3}$ material (the value of refractive index and extinction) as the function of the absorbed volumetric charge which is changing together with the concentration of the absorbed gas. In the paper, the parameters of the material were defined for a wide wavelength range $(300-2500 \mathrm{~nm})$. In the course of the calculations, the values corresponding to the wavelength of $650 \mathrm{~nm}$ were applied (Fig. 5). During the numerical simulations, the refractive index was being changed within 1.94-1.8. For the needs of the numerical analysis, in order to obtain denser changes of the refractive index and extinction, the above points were interpolated with the third order polynomial.

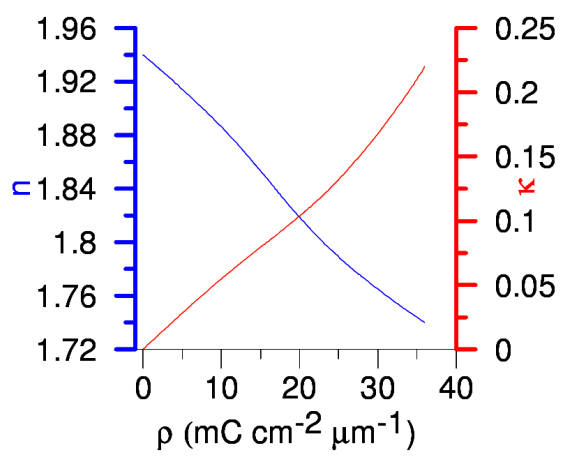

Fig. 5. Parameters of $\mathrm{WO}_{3}$ as a function of the absorbed volumetric charge [7].

\subsubsection{Design}

It is important to select an appropriate geometry and structure. Particularly critical was the choice of sensor layer thickness. Due to a relatively high value of the extinction factor of the sensor layer, the light was strongly attenuated already on short propagation sections. It is possible to select the thickness of the sensor layer small enough to ensure that the modes are excited only in the multimode core (Fig. 6). In this case, the interaction is taking place through the evanescent field of modes excited in the multimode core. In the case of thicker layers, the modes will also be excited in the sensor layer. In this case, the value of attenuation of the propagating light in the core can be selected by influencing the overlap of the wave functions excited in the sensor layer and in the core. In the figure we marked the fragments of the wave functions of the modes whose energy is located mainly in the core. The marked fragments correspond to the evanescent field of a given mode which is propagating in the sensor layer. As it can be observed, the higher the marked peak is, the larger part of the energy penetrates into the sensor layer. We can see that a slight change of cover thickness, through influencing the character of wave functions of the modes, has a significant influence on the obtained results.
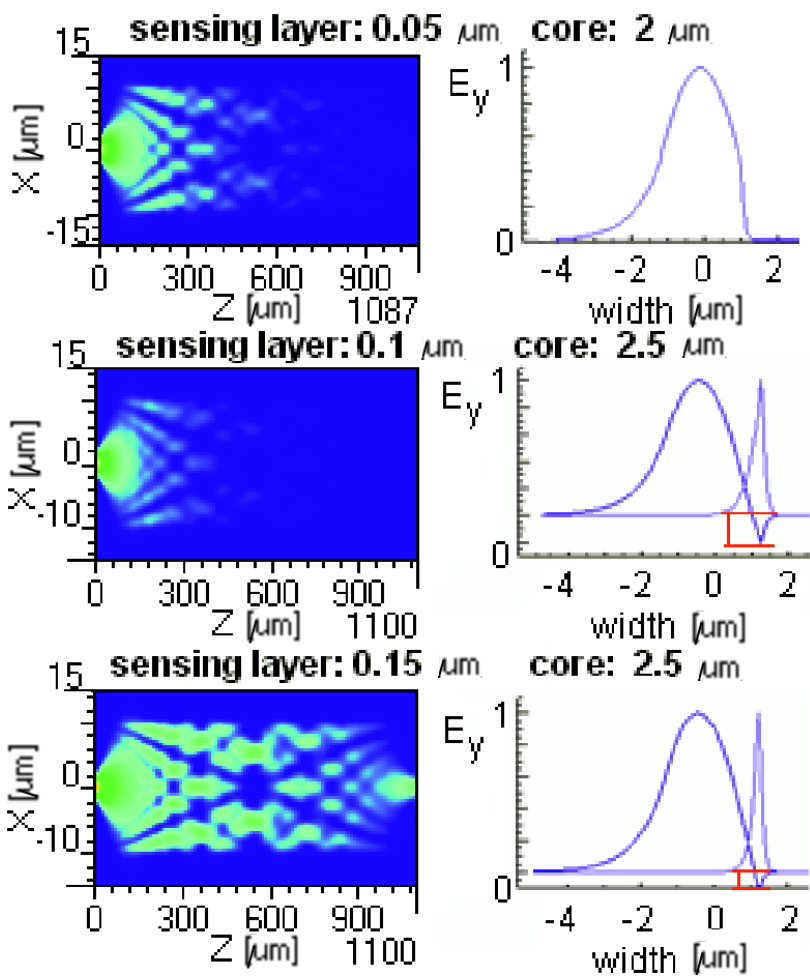

Fig. 6. Field distributions in the multimode section (width $20 \mu \mathrm{m}$ ) and corresponding to them wave functions of modes excited in the waveguides for definite parameters.

\subsubsection{Results and discussions}

Basing on the modal analysis, we can select a geometry of the structure which can ensure the acquisition of the expected amplitude dependence on the refractive index of the sensor layer. Figure 7 presents the dependence of the output field amplitude as a function of optical parameters of the sensor layer. The calculations were carried out for the structures of the sensor layer thickness of $0.15 \mu \mathrm{m}$ and 
$0.05 \mu \mathrm{m}$. The length of the structure was selected in the way to ensure that when there is no absorbed volumetric charge at the output of the multimode section, we obtain 1-fold image of the input field.

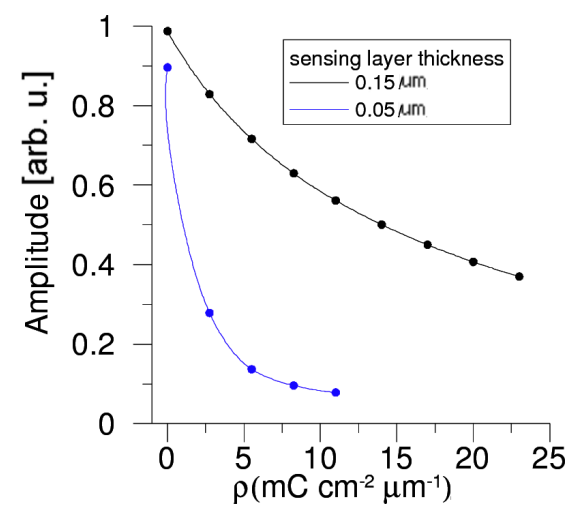

Fig. 7. Output field amplitude as a function of sensor layer parameters.

In this case the oscillations which could be seen in Fig. 5 are not occurring on the amplitude characteristic. Those oscillations were affected by the coupling of modes from the sensor layer and from the core. When the light is strongly attenuated in the sensor layer, the distribution of the field in this layer is the reflection of the distribution in the core. Due to strong attenuation, the effects of the intermodal interference in the sensor layer alone are less visible.

\subsection{Mach-Zehnder interferometer}

The Mach-Zehnder interferometer is a device used to determine the relative phase shift between two collimated beams from a coherent light source [9].

\subsubsection{Operation principle}

The model of the analyzed device was presented in Fig. 8. In this case, the MMI section ( $51 \mu \mathrm{m}$ wide) made up one of the arms of the Mach-Zehnder interferometer. The second arm was made up by a single mode waveguide (4 $\mu \mathrm{m}$ wide).

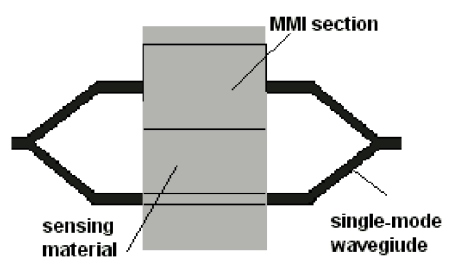

Fig. 8. M-Z interferometer whose one arm was made up by a multimode section and the second arm by a single mode waveguide.

The field distribution at the distance $z$ from the beginning of MMI section is described by the following relation:

$$
E(x, y, z)=\exp \left(-\mathrm{j} \beta_{0} z\right) \sum_{l} c_{l} \varphi_{l}(x, y)
$$

$$
\times \exp \left(-\mathrm{j}\left(\beta_{0}-\beta_{l}\right) z\right) .
$$

At the distance $z$ corresponding with the coupling, the equation assumes the following form:

$$
\begin{aligned}
& E(x, y, z)=\exp \left(-\mathrm{j} \beta_{0} z\right) \sum_{l} c_{l} \varphi_{l}(x, y) \\
& \quad=\exp \left(-\mathrm{j} \beta_{0} z\right) E(x, y, 0) .
\end{aligned}
$$

Then, the signal phase after covering the distance equal to the coupling path will be corresponding with the phase of a base mode of the multimode waveguide. The phase difference $\Delta \Phi$ at the ends of the multimode waveguide and single mode waveguide would be therefore dependent on the difference between propagation constants of the base mode of the multimode waveguide $\beta_{10}$ and single mode waveguide $\beta_{20}$ according to the equation

$$
\Delta \Phi=d\left(\beta_{10}-\beta_{20}\right) .
$$

Hence the idea arises to design the Mach-Zehnder interferometer whose one arm was made up by a multimode section of the length equal to the coupling path, and the other one by a single mode waveguide. The detection method of the proposed sensor was based in this case on the measurement of phase difference of waves propagating in the arms of the interferometer.

\subsubsection{Design}

The multimode waveguide had the length equal to the coupling path of the MMI structure for 1-fold images. The analysis was carried out for the wavelength of $1310 \mathrm{~nm}$, refractive index of the core 1.5, refractive index of the substrate 1.49. The refractive index was being changed within 1.47-1.49. The length of the multimode section was selected in the way to ensure that for the refractive index of 1.47 we could obtain the image of the input field at the output.

\subsubsection{Results and discussions}

The lengths, thicknesses of particular layers as well as the cover of both waveguides are the same. Yet the phase changes in the multimode section and in the single mode waveguide are totally different (Fig. 9).

The values of phase difference at the outputs of MMI section and single mode waveguide in the function of refractive index of the cover layer are presented in Fig. 10.

The difference in phases at the outputs of the investigated waveguides is changing with the change of the refractive index of the cover. When designing a sensor based on the Mach-Zehnder interferometer whose one arm is made up by a single mode waveguide and the other one by a multimode section, we can have influence on its sensitivity by changing the width of the MMI section. A good advantage of this configuration, in contrast to standard ones, is the possibility to cover both arms with material sensitive to the changes of the parameters of the environment. The sensor layer can cover the whole structure of the interferometer, and the arms can be placed at short distance. It considerably simplifies the technological process. 

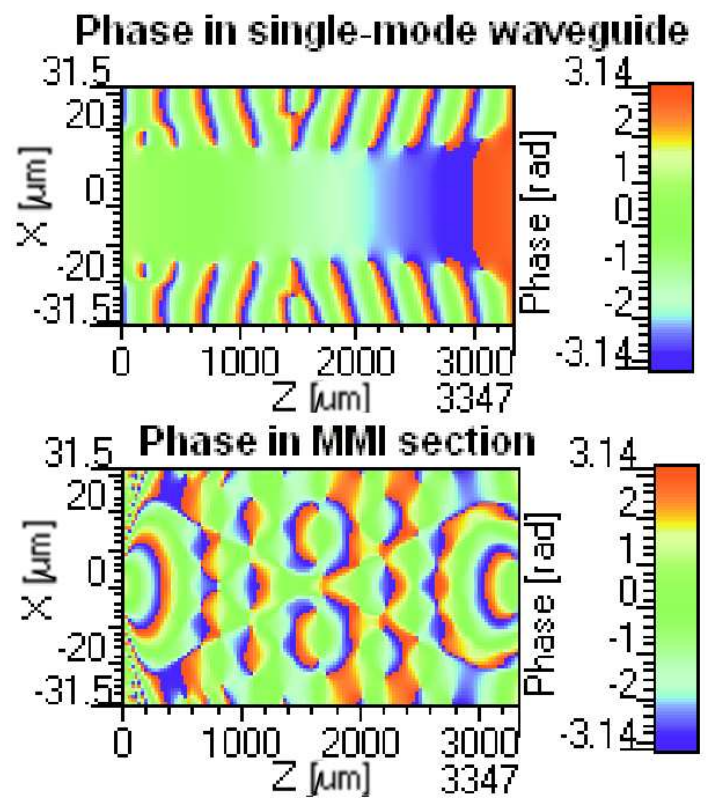

Fig. 9. Phase along the propagation path for the single mode waveguide (a) and multimode section (b).

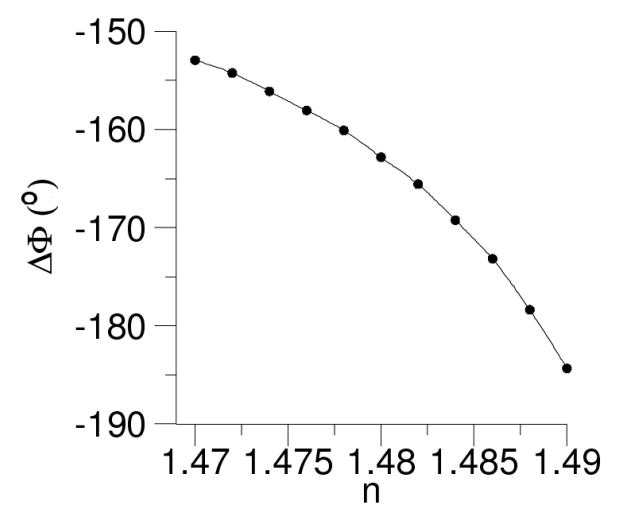

Fig. 10. Phase difference at the ends of $\mathrm{M}-\mathrm{Z}$ interferometer in the function of refractive index of the cover.

\section{Conclusions}

The discussed concepts can be easily adapted to various applications by the selection of an appropriate sensor material and choice of suitable dimensions of the multimode waveguide. The sensitivity can be increased through the change of layer thickness and the width of the multimode waveguide.
In the case of the first investigated configuration, we obtain high change dynamics of the output amplitude in the function of refractive index of the cover. This configuration is advantageous when high sensitivity systems have to be applied. When the cover is characterized by non-zero extinction value, the dependence of the output signal in the function of cover layer parameters does not have an oscillating character. Here, the shape and sensitivity of the characteristic can be modified by influencing the form of wave functions propagating in the sensor layer of the modes. The detection method is most comfortable in the case of the Mach-Zehnder interferometer for which the differences of phases are measured.

\section{Acknowledgments}

The work was sponsored by the State Committee for Scientific Research (NCBiR) within the grant N R01 034 06/2009.

\section{References}

[1] R. Marz, Integrated Optics: Design and Modeling, New York 1995.

[2] K. Chen, C. Leung, I. Chang, Opt. Lett. 15, 582 (1990).

[3] M. Błahut, D. Kasprzak, M. Sujewicz, Acta Phys. Pol. A 116, 261 (2009).

[4] K.R. Kribich, R. Copperwhite, H. Barry, B. Kolodziejczyk, J.M. Sabattie, K. O'Dwyer, B.D. MacCraith, Sensors Actuators $B$ 188, 881 (2005).

[5] T. Pustelny, M. Grabka, M. Błahut, D. Kasprzak, Acta Phys. Pol. A 116, 385 (2009).

[6] T. Mazingue, R.K. Kribich, P. Etienne, Y. Moreau, Opt. Commun. 278, 312 (2007).

[7] L.B. Soldano, E.C.M Pennings, Lightwave Technol. 13, 615 (1995).

[8] K. von Rottkay, M. Rubin, S.-J. Wen, Thin Solid Films 306, 10 (1997).

[9] A. Irace, G. Breglio, Opt. Exp. 11, 2807 (2003).

[10] K. Gut, J. Phys. (France) 87, 79 (2006).

[11] M. Błahut, D. Kasprzak, Acta Phys. Pol. A 116, 257 (2009).

[12] E. Mach, The Principles of Physical Optics, Berlin 2003, p. 170. 